\section{Une résistance à l'hormone anti-müllérienne, due à une mutation du gène de son récepteur, est responsable de certains cas de syndrome de persistance des canaux de Müller}

L'hormone anti-müllérienne (AMH) est le facteur testiculaire responsable de la régression des canaux de Müller chez le foetus mâle. Produite par les cellules de Sertoli [1], c'est un dimère appartenant à la superfamille du transforming growth factor TGF $\beta$. Les équipes de l'Unité 293 de l'Inserm (ENS, Montrouge) et de la société Biogen (Cambridge, USA) qui avaient, chacune de leur côté, cloné l'AMH voici 10 ans, ont conjugué leurs efforts pour cloner en 1994 l'ADNc récepteur de l'AMH chez le lapin [2] en utilisant son homologie avec les récepteurs déjà clonés du TGFß [3] et de l'activine [4]. Il s'agit d'une nouvelle famille de récepteurs, des sérine/thréonine kinases possédant un seul domaine transmembranaire. Dans le numéro de décembre 95 de la revue Nature Genetics, ces équipes rapportent le clonage de l'ADNc et du gène humains codant pour le récepteur de l'AMH [5].

Il s'agit du récepteur de l'AMH de type II, considéré comme le récepteur primaire [6], qui est capable de lier l'hormone, mais qui a besoin d'un récepteur de type I - qui est peut-être le substrat de son activité kinase - pour pouvoir transduire le signal.

Le gène codant pour le récepteur de l'AMH a une longueur de $8 \mathrm{kpb}$, contient 11 exons (figure $1 \mathrm{~A}$ ) et est localisé au début du bras long du chromosome 12, sur la bande 12q13. Son messager, d'environ 2050 bases, code pour une protéine de 573 acides aminés (figure $1 B$ ). Entre autres éléments régulateurs potentiels, le promoteur du récepteur comporte un élément de réponse au facteur stéroïdogénique SF1 $\left(\mathrm{m} / \mathrm{s} n^{\circ} 10\right.$, vol. 10, p. 1054) [7], qui pourrait être fonctionnel car il est conservé dans le promoteur de la souris. Cet élément de réponse existe aussi dans le promoteur du gène $A M H$, ce qui pourrait expliquer le fort parallélisme existant entre l'expression de l'hormone et celle de son récepteur: tous deux sont produits à la fois par le testicule fotal et postnatal, et par l'ovaire adulte. En revanche, et comme il fallait s'y attendre, le récepteur est synthétisé dans les organes cibles de l'AMH, canal de Müller et ovaire fotal, mais pas l'hormone.

La preuve définitive de l'identité de ce récepteur est apportée par son implication dans un cas de syndrome de persistance des canaux de Müller (PMDS). Également appelé syndrome de «l'homme à utérus», il s'agit d'une forme peu fréquente de pseudo-hermaphrodisme masculin consistant en la présence d'un utérus et/ou de trompes chez des garçons dont les organes génitaux externes sont normalement virilisés, le signe d'appel étant la cryptorchidie ou la présence d'une hernie inguinale. Un grand nombre de mutations avaient déjà été identifiées dans le gène codant pour l'AMH [8], mais plus de la moitié des patients PMDS étudiés avaient un taux normal d'AMH sérique, et avaient un gène normal. Ils pouvaient donc être de bons candidats pour des mutations du gène codant pour le récepteur. Chez l'un d'entre eux, en particulier, une biopsie effectuée pour vérifier l'état du testicule avant son abaissement chirurgical avait confirmé la présence d'AMH biologiquement active.

C'est chez ce patient que la première mutation a été identifiée, à l'état homozygote, dans les séquences codant pour la partie extracellulaire du récepteur, responsables donc de la fixation du ligand. Il s'agit d'une transition $G \rightarrow A$ de la première base invariante du site donneur d'épissage du second intron (figure 1C). Fait intéressant, cette mutation provoque un épissage alternatif avec formation de deux messagers anormaux: (1) un messager court résultant du saut de l'exon 2 avec épissage direct des exons 1 et 3 . Un tel messager existe naturellement chez le lapin, où il ne permet pas la production d'un récepteur fonctionnel [2] ; (2) un messager conservant un exon 2 rallongé de 12 bases, par suite de l'utilisation d'un site cryptique donneur d'épissage situé 12 bases plus en aval dans l'intron 2. Ce messager code pour cinq acides aminés anormaux: une glycine remplaçant une asparagine, suivie de quatre acides aminés surnuméraires. Pour l'étude du mode d'action des récepteurs du TGF $\beta$ et de l'activine, beaucoup de mutations artificielles ont été produites dans des souches cellulaires, aucune mutation naturelle n'ayant encore été observée. La mutation du récepteur de l'AMH rapportée dans Nature Genetics est la première mutation naturelle mise en évidence dans cette nouvelle famille de récepteurs.

Il reste à rechercher l'existence effective d'un récepteur de type I pour 


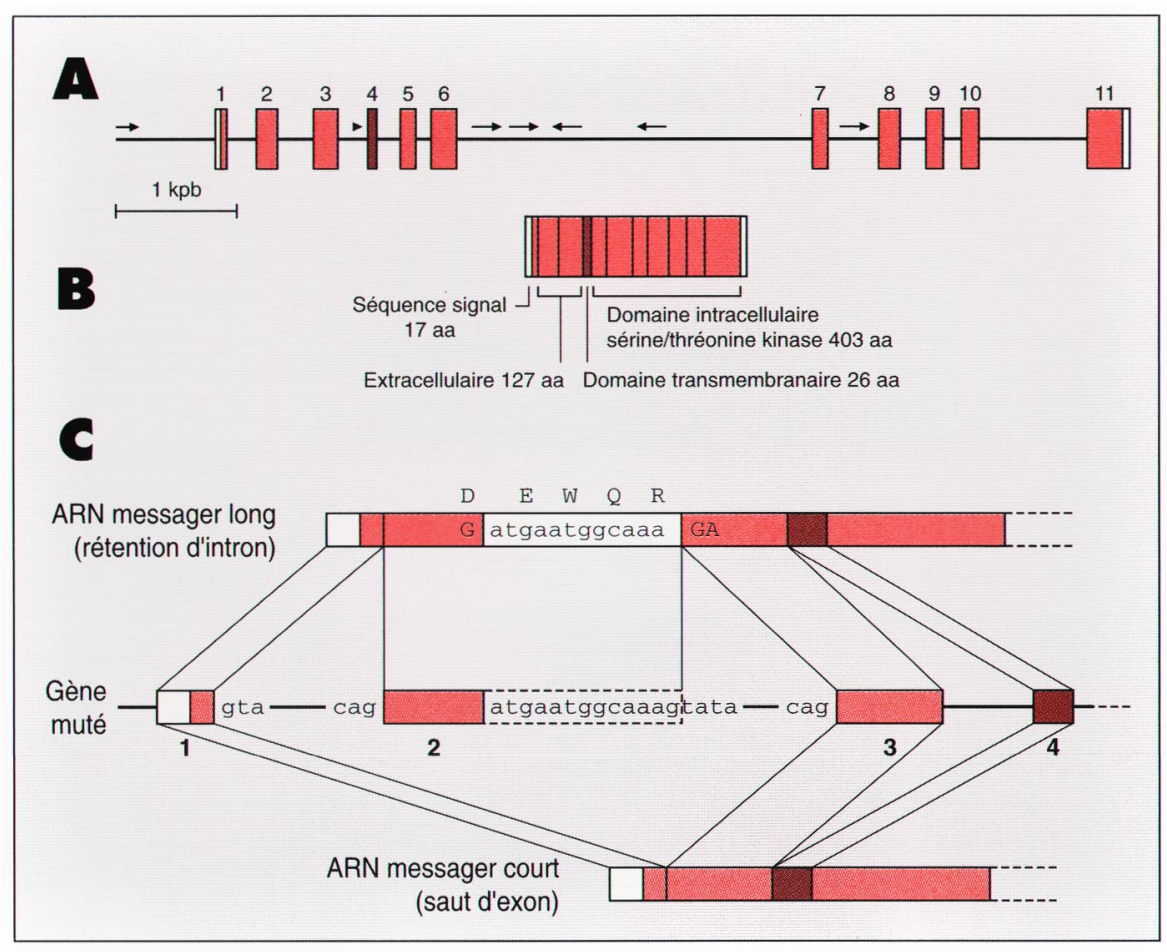

Figure 1. Le gène humain du récepteur de l'AMH. A: Vue générale du gène. Mesurant plus de $8 \mathrm{kpb}$, il est organisé en 11 exons. Les flèches représentent la position de séquences Alu, présentes notamment dans le grand intron central. B: Schéma de I'ARNm, avec indication de la taille des différents domaines de la protéine pour laquelle il code. C: Mutation du gène codant pour le récepteur chez un "homme à utérus". La mutation de la première base d'un site donneur d'épissage produit par épissage alternatif deux messagers anormaux.

l'AMH. L'étude génétique des autres patients PMDS devrait montrer si des mutations de l'ADNc du récepteur de type II qui vient d'être cloné peuvent rendre compte de tous les cas de PMDS avec gène $A M H$ normal. Dans le cas contraire, il conviendra de rechercher des mutations dans le récepteur de type I. Il est cependant possible que les récepteurs de type I puissent avoir une moins grande spécificité, et s'associer à différents récepteurs de type II; il ne serait alors pas impossible qu'une mutation d'un récepteur de type I puisse avoir un effet létal.

J.-Y.P.

1. Josso N, Rey R. La cellule de Sertoli, une cellule
2. di Clemente N, Wilson CA, Faure E, Boussin L, Carmillo P, Tizard R, Picard JY, Vigier B, Josso N, Cate RL. Cloning, expression and alternative splicing of the receptor for anti-Müllerian hormone. Mol Endocrinol 1994; 8 : 1006-20.

3. Lin HY, Wang XF, Ngeaton E, Weinberg RA, Lodish HF. Expression cloning of the TGF- $\beta$ type II receptor, a functional transmembrane serine/threonine kinase. Cell 1992; 68: 775-85.

4. Mathews LS, Vale WW. Expression cloning of an activin receptor, a predicted transmembrane serine kinase. Cell 1991 ; 65 : 973-82.

5. Imbeaud S, Faure E, Lamarre I, Mattéi MG, di Tragethon L, Tonkin C, Nelson J, McAuliffe M, Bidart JM, Lababidi A, Josso N, Cate RL, Picard JY. Insensitivity to anti-Müllerian hormone due to a spontaneous mutation in the human anti-Müllerian hormone receptor. Nature Genet 1995; 11: 382-7.

6 . Massagué J. Receptors for the TGF- $\beta$ family. Cell 1992 ; 69: 1067-70.

7. Shen WH, Moore CCD, Ikeda Y, Parker KL, Ingraham HA. Nuclear receptor steroidogenic factor 1 regulates the mullerian inhibiting substance gene : a link to the sex determination cascade. Cell 1994; $77: 651-61$.

8. Imbeaud S, Carré-Eusèbe D, Rey R, Belville C, Josso N, Picard JY. Molecular genetics of the persistent Müllerian duct syndrome: a study of 19 families. Hum Mol Genet 1994; 3 : 125-31. Clemente N, Tizard R, Carré-Eusèbe D, Belville C,

\section{BRÈVES}

Migraine. Nous avons rapporté dans nos colonnes $(\mathrm{m} / \mathrm{s}$ $n^{\circ} 11$, vol. 9, p. 1287) la localisation en 19 p13 d'un gène impliqué dans la migraine familiale hémiplégique par une équipe française [1]. Il s'agit d'une forme très rare de migraine, avec aura et hémiparésie unilatérale transitoire, dont la transmission autosomique dominante est évidente. Le problème des autres formes de migraine commune, où des facteurs génétiques semblent assez souvent impliqués, reste entier malgré les nombreux efforts faits pour mieux comprendre cette maladie si fréquente. Une étude épidémiologique [2] a montré, en effet, que les migraines affectent $8 \%$ des hommes et $25 \%$ des femmes de la population générale. Deux équipes, l'une allemande et l'autre hollandaise, ont uni leurs efforts pour rechercher une liaison avec ce locus en 19p13 dans 28 familles où plusieurs sujets de la même fratrie étaient atteints de migraine avec ou sans prodromes [3]. L'analyse de liaison, réalisée avec un seul marqueur polymorphique (D19394), et calculée à l'aide de deux méthodes statistiques, semble positive, du moins dans quelques familles. Néanmoins, étant donné l'extrême probabilité de l'hétérogénéité génétique et la multiplicité des facteurs intervenant dans les migraines, de nombreux travaux seront encore nécessaires et il serait prématuré de conclure pour l'instant.

[1. Joutel A, et al. Nature Genet $1993 ; 5: 40-5$.]

[2. Rasmussen BK, et al. J Clin Epidemiol 1991 ; 44: 1147-57.]

[3. May A, et al. Hum Genet 1995; 96: 604-8.] 